\title{
A class of replacement systems with simple optimality theory
}

\section{John Staples}

\begin{abstract}
A class of replacement systems is studied which satisfies a
"subcommutativity" condition. Examples of systems satisfying this condition are many of the systems of graph-like expressions which have recently been studied in connection with the efficient evaluation of recursive definitions. An optimality theory of subcommutative systems is developed and is used to give conditions which are sufficient to ensure that an evaluation (reduction) procedure is optimal. The optimality theory is also applied to develop conditions under which a given subcommutative system speeds up, in a natural sense, another replacement system.
\end{abstract}

\section{Introduction}

It has been understood for several years (see for example [9]) that efficient evaluation of expressions is often facilitated by graph-like representations of the expressions. For particular simple systems of graph-like expressions, for example $[2,3,8]$, certain outermost evaluation procedures have been noticed to be optimal. In this paper the underlying structure which is the source of this increased efficiency is exposed through a generalisation of the theory to abstract replacement systems.

This generalisation is being used by the author in a study of optimal evaluation algorithms for systems of graph-like expressions; see for example [7]. It promises to be applicable also to the still more general, category-theoretic, approach to graph-like expressions which is being undertaken by Ehrig and Rosen in, for example, [1].

Received 31 May 1977. 


\section{Subcommutative systems}

2.1. We consider replacement systems $(B, \rightarrow)$ in the sense of Rosen [4]. That is, $B$ is a set and $\rightarrow$ is a subset of $B \times B$. We write $a \rightarrow b$ to denote that $(a, b) \epsilon \rightarrow$, and in that case say that $a$ contracts to $b$, and that $a \rightarrow b$ is a contraction. A sequence

$$
a_{1} \rightarrow a_{2}+\ldots+a_{n}, n \geq 1 \text {, }
$$

of contractions is called a reduction from $a_{1}$ to $a_{n}$ and may be indicated briefly by $a_{1} \rightarrow^{*} a_{n}$. The number of contractions in a reduction is called the length of the reduction. If $a \in B$ is such that there is no $b$ such that $a \rightarrow b$ then $a$ is said to be in normal form. If $a \rightarrow^{*} b$ where $b$ is in normal form then $a$ is said to have the normal form $b$. We write $B_{N}$ for the set of $a \in B$ which have normal forms. If $a \in B_{N}$, then there is a least number $n \geq 0$ which is the length of some reduction of $a$ to some normal form; we denote this least number $|a|$, and may call it the norm of $a$.

2.2. We call a replacement system $(B, \rightarrow)$ subcommutative if for all $a \in B_{N}$ and all $a \rightarrow b, a \rightarrow c$, either

$$
\begin{aligned}
& \text { (i) } b=c \text {, or } \\
& \text { (ii) } b \rightarrow c \text {, or } \\
& \text { (iii) } c \rightarrow b \text {, or } \\
& \text { (iv) there is } d \rightarrow B \text { such that } b \rightarrow d \text { and } c \rightarrow d \text {. }
\end{aligned}
$$

A published example of a system with this property is given in Pacini [2] and in Pacini et al [3]. The discussion of it given there appears technically inadequate, but is nevertheless stimulating.

2.3. Since the above definition of subcommutativity places restrictions only on points $a \in B_{N}$, such systems need not have the Church. Rosser property; that is they need not satisfy the condition: for all $a \rightarrow^{*} b, a \rightarrow^{*} c$, there is $d \rightarrow B$ such that $b \rightarrow^{*} d, c \rightarrow^{*} d$. In particular, any replacement system without any normal forms is subcommutative. 
2.4. We shall see however that subcommutativity is enough to give the normal form theorem for $(B, \rightarrow)$; that is, every $a \in B_{N}$ has just one normal form. It will also be made clear that, writing $\rightarrow_{N}$ for the restriction of $\rightarrow$ to $B_{N} \times B_{N}$, that ${ }_{N}$ is just

$$
\left\{(a, b) \in \rightarrow: a \in B_{N}\right\} \text {, }
$$

and that $\left(B_{N}, \rightarrow_{N}\right)$ has the Church-Rosser property.

\section{The normal form theorem and related results}

Throughout this section we assume that $(B, \rightarrow)$ is subcomutative.

3.1. The key lemma is :

If $a \in B_{N}$ has a reduction $R$ of length $n$ to normal form $f$, and if $a \rightarrow b$, then $b$ has a reduction to $f$, so in particular $b \in B_{N}$.

Proof. By induction on $n$. The case $n=0$ is vacuously true, so suppose that $n>0$. Say that the first contraction of $R$ is $a \rightarrow a^{\prime}$. If $a^{\prime}=b$ or $b \rightarrow a^{\prime}$ then it is clear that $b$ has a reduction to $f$. If $a^{\prime} \rightarrow b$ then the inductive hypothesis applies to $a^{\prime}$ to show that $b$ has a reduction to $f$. The remaining possibility is that there is $d \in B$ such that $a^{\prime} \rightarrow d$ and $b \rightarrow d ;$ in that case the inductive hypothesis applies to $a^{\prime}$ to show that $d$ has a reduction to $f$, so as $b \rightarrow d, b$ has a reduction to $f$.

3.2. We now define a binary relation $\rightarrow_{\leq 1}$ on $B$ by: $a \rightarrow_{\leq 1} b$ if and only if $a=b$ or $a \rightarrow b$. We can now show the

3.3. Church-Rosser Theorem for $\left(B_{N}, \rightarrow_{N}\right)$. If $a \rightarrow_{N}^{*} b, a \rightarrow_{N}^{*} c$, then there is $d \in B_{N}$ such that $b \rightarrow_{N}^{*} d, c \underset{N}{*} d$.

It is immediate from 3.1 and the definition of subcommutativity that if $a \in B_{N}$ and $a \rightarrow_{\leq 1} b, a \rightarrow_{\leq 1} c$, then $b, c \in B_{N}$ and there is $d$ such that $b \rightarrow_{\leq 1} d, c \rightarrow_{\leq 1} d$. As $\rightarrow_{N} \leq \rightarrow_{\leq 1}$, the Church-Rosser Theorem follows by a standard (Rosen [4]) and elementary argument.

As usual, the normal form theorem for $\left(B_{N}, \rightarrow_{N}\right)$ is a corollary of the 
Church-Rosser Theorem, so we have the

3.4. Normal form theorem for $(B, \rightarrow)$. If $a \in B_{N}$ then a has just one normal form.

Proof. Any two reductions in $(B, \rightarrow)$ of $a \in B_{N}$ to normal forms are evidently re?...tions in $\left(B_{N}, \rightarrow_{N}\right)$ to normal forms, so the normal form theorem for $\left({ }_{N}{ }_{N} \rightarrow{ }_{N}\right)$ applies to assert that the two normal forms are the same.

\section{Optimal and dummy contractions in subcommutative systems}

4.1. The results of this section up to and including 4.3 apply to any replacement system which has the normal form property, and property 3.1 .

If $a \in B_{N}$ and $a \rightarrow b$, we may write $a \rightarrow_{0} b$ and call $a \rightarrow b$ an $o$-contraction if there is a reduction of $a$ to normal form of length $|a|$ whose first contraction is $a+b$. If $a \rightarrow b$ is not an o-contraction we may write $a+\delta b$ and may call $a+b$ a dummy contraction. We may also write $a \rightarrow_{0}^{*} b$ and $a \rightarrow_{\delta}^{*} b$ to denote reductions comprising o-contractions and dummy contractions respectively.

The following lemma is basic.

4.2. If $a \in B_{N}$ and $a \rightarrow b$ then

(i) $|a| \leq|b|+1$,

(ii) $a \rightarrow_{0} b$ if and only if $|a|=|b|+1$,

(iii) $a \rightarrow_{\delta} b$ if and only if $|a| \leq|b|$,

(iv) there is $c$ such that $a \rightarrow_{0} c$.

Proofs. (i) is clear, since from 3.1, $b \in B_{N}$, and $a \rightarrow b$ followed by a reduction of $b$ to normal form of length $|b|$ is a reduction of $a$ to normal form.

(ii) is also elementary. If $a \rightarrow_{0} b$ then $a \rightarrow b$ begins a reduction $R$ of $a$ of length $|a|$ to normal form, and the remainder of $R$ 
constitutes a reduction of $b$ to normal form which must be of minimal length (or else $R$ is not a reduction of a to normal form of minimal length). That is,

$$
|a|=|b|+1 \text {. }
$$

If conversely $|a|=|b|+1$, then no reduction of $a$ to normal form has a length which is shorter than the reduction which begins $a \rightarrow b$ and concludes with a reduction of $b$ to normal form of length $|b|$; hence by definition of o-contraction, $a+0 b$.

(iii) is immediate from $(i)$ and $(i i)$.

(iv) is immediate from the definition of o-contraction.

4.3. It follows immediately from 4.2 (ii) that the o-reductions of $a$ of length $|a|$ are just the reductions of $a$ to normal form of minimal length.

The next result, which is basic to what follows, makes use of the subcommutativity hypothesis.

4.4. If $a \in B_{N}$ and $a \rightarrow b$ then $a \rightarrow_{\delta} b$ if and only if $|a|=|b|$.

Proof. One part is clear from 4.2 (iii), so we argue by induction on $|a|$ that if $a{ }_{\delta} b$ then $|a|=|b|$. The case $|a|=0$ is vacuously true so we suppose that $|a|>0$. Then tinere is $c$ such that $a \rightarrow_{0} c$, so that $|c|=|a|-1$ and $b \neq c$. Moreover it is not the case that $c+b$, for that would imply either $c \rightarrow_{0} b$, when $|b|=|a|-2$, contradicting $a \rightarrow b$, or else $c{ }_{\delta} b$, when by inductive hypothesis $|b|=|c|=|a|-1$, so that $a \rightarrow_{0} b$, a contradiction. Hence in view of subcommutativity there are two remaining cases, as follows.

CASE 1. $b \rightarrow c$. Then $|b| \leq|c|+1=a$, so from 4.2 (iii), $|a|=|b|$.

CASE 2. There is $d$ such that $b \rightarrow d, c \rightarrow d$. It is not the case that $c \rightarrow_{0} d$, or else $|d|=|a|-2$, so that $a \rightarrow_{0} b \rightarrow_{0} d$, contradicting $a+{ }_{\delta} b$; hence $c \rightarrow_{\delta} d$. Then by inductive hypothesis $|d|=|c|=|a|-1$. Thus it is not the case that $b{ }_{\delta} d$, or else from 
4.2 (iii), $|b| \leq|d|=|a|-1$, so applying 4.2 (iii) again, $|a| \leq|b| \leq|a|-1$, a contradiction. So $b \rightarrow_{0} d$, so

$|b|=|a|+1=|a|$, as required.

\section{A practicable sufficient condition for optimality of a reduction procedure}

5.1. Although the foregoing provides a convenient framework for discussion of optimal reductions, by itself it does little to help recognize optimal reductions in practice. A typical practical problem is: given $\rightarrow_{u} \subseteq \rightarrow$, give practicable conditions which are sufficient to ensure that $\rightarrow_{u}$ is optimal. That is, the conditions should ensure that $\rightarrow_{u}$ is both

(i) complete; that is if $a \in B_{N}$ then $a \rightarrow_{u}^{*} f$ where $f$ is in normal form; and

(ii) minimal; that is if $a \rightarrow * f$ has length $n$, where $f$ is in normal form, then every reduction $\underset{u}{a} f$ has length at most $n$.

The following result is suitable for the purpose.

5.2. If $(B, \rightarrow)$ is a subcommutative system, if $\rightarrow_{u} \subseteq \rightarrow$, and if $\rightarrow_{u}$ satisfies the following conditions, then $\rightarrow_{u}$ is optimal:

(i) for all $a \in B_{N}$ such that $a$ is not in normal form, there is $b$ such that $a \rightarrow_{u} b$,

(ii) whenever $a \in B_{i V}, a \rightarrow_{u} b$ and $a \rightarrow c$ there is $d$ such that $c \rightarrow^{*} d, b \rightarrow^{*} d$, and if $b \rightarrow^{*} d$ comprises $k$ contractions then $c \rightarrow * d$ includes at least $k$ contractions from $\rightarrow u$.

Proof. We prove by induction on $|a|$ that if $a \in B_{N}$ and $a \rightarrow_{u} b$ then $a \rightarrow_{0} b$.

The case $|a|=0$ is vacuously true, so suppose that $|a|>0$. There is $c$ such that $a \rightarrow_{0} c$. From (ii) there is $d$ such that 
$b \rightarrow^{*} d, c \rightarrow^{*} d$ and, writing $k$ for the length of $b \rightarrow^{*} d, c^{*} d$ includes at least $k$ contractions from $\rightarrow_{u}$. Since $|c|<|a|$ it follows from 4.2, 4.4, and the inductive hypothesis that all $u$-contractions in $c \rightarrow^{*} d$ are o-contractions, so $|a| \leq|a|-k-1=|a|-(k+1)$. Now $a \rightarrow_{u} b \rightarrow^{*} d$ is a reduction of length $k+1$, so all of its contractions are o-contractions; in particular, $a \rightarrow_{0} b$ as required.

The result 5.2 is applied to a theory of graph-like expressions in $[7]$.

\section{Normal representations and speedups}

6.1. In this section we define notions of normal representation of a system $\underline{\underline{B}}$ by a system $\underline{\underline{C}}$, and notions of speedup of a system $\underline{B}$ by a system $\stackrel{\underline{C}}{ }$, and we give some of their elementary properties.

All the systems to be studied in this and the following sections are assumed to have the normal form property. The hypothesis of subcommutativity will be mentioned when it is required.

Given a replacement system $\underline{\underline{B}}=(B,+)$, we may write $n(a)$ for the normal form of an element $a$ of $B$. Since this notation does not specify the system $B$ with respect to which $n(a)$ is the normal form of $a$, it is potentially ambiguous; but the ambiguity will not cause confusion here.

6.2. A weak normal representation $S$ of a system $\underline{\underline{B}}=(B, \rightarrow)$ by a system $\cong=(C, \rightarrow)$ is a relation $S \subseteq B_{N} \times C_{N}$ such that:

(i) for all $b \in B_{N}$ there is $c \in C_{N}$ such that $(b, c) \in S$;

(ii) for all $(b, c) \in S,(n(b), n(c)) \in S$ (see Figure 1 ).

Evidently $S$ can be regarded as a multivalued function from $B_{N}$ to $c_{N}$

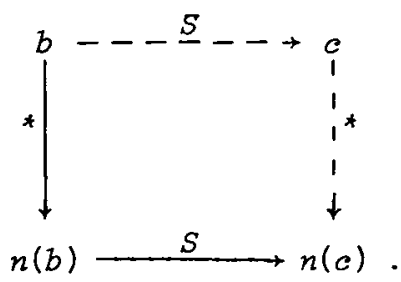

FI GURE 1 
We may say, when $(b, c) \in S$, that $c$ represents $b$. Note that if $S$ is a weak normal representation and $b \in B_{N}$ then we can calculate a representation of $n(b)$ in the system $\underset{C}{C}$ by choosing $c$ such that $(b, c) \in S$ and calculating $n(c)$. However we do not generally have the means to recover $n(b)$ from $n(c)$; hence the following stronger notion.

6.3. A normal representation $S$ of $B$ by $\underset{\sim}{\mathrm{C}}$ is a weak normal representation which also has the property:

for alz $c^{\prime} \in C_{N}$ there is at most one $b^{\prime} \in B_{N}$ such that for some $(b, c) \in S, b^{\prime}=n(b), c^{\prime}=n(c)$.

Notice that if $S$ is a normal representation and $b \in B_{N}$ then we can calculate $n(b)$ by computing in $C$; that is, choose $c$ such that $(b, c) \in S$, reduce $c$ to $n(c)$, and then recover $n(b)$ as the unique element $b^{\prime \prime}$ such that $\left(b^{\prime \prime}, n(c)\right) \in S$.

6.4. In applications normal representations may have an additional property as follows.

A strong normal representation $S$ of $\underline{\underline{B}}$ by $\stackrel{\mathrm{C}}{=}$ is a normal representation such that for all $c \in C_{N}$ there is exactly one $b \in B_{N}$ such that $(b, c) \cdot \in S$.

Each of the above notions of normal representation leads to a corresponding notion of speedup, as follows.

6.5. A weak speedup (respectively speedup, strong speedup) $S$ of $\underline{B}$ by $\stackrel{C}{=}$ is a weak normal representation (respectively normal representation, strong normal representation) such that for all $(b, c) \in S$,

$$
|c| \leq|b| \text {. }
$$

6.6. It is convenient for applications to notice that the above notions of normal representation and speedup are transitive. Indeed, given systems $\underline{B}=(B, \rightarrow), \underline{\underline{C}}=(C, \rightarrow), \underline{\underline{D}}=(D, \rightarrow)$, and relations $S \subseteq B_{N} \times C_{N}, S^{\prime} \subseteq C_{N} \times D_{N}$, we denote by $S^{\prime} \circ S$ the relation

$$
\begin{aligned}
S^{\prime} \circ S & =\left\{(b, d): \text { for some } c \in C,(b, c) \in S, \text { and }(c, d) \in S^{\prime}\right\} \\
& \subseteq B_{N} \times D_{N},
\end{aligned}
$$

and we have: 
6.7. If $S, S^{\prime}$ are both weak normal representations (respectively normal representations, strong normal representations, weak speedups, speedups, strong speedups) then so is $S^{\prime} \circ S$.

Proof. Each of the conditions of 6.2 (i), 6.2 (ii), 6.3,6.4, and 6.5 follows for $S^{\prime} \circ S$ from the same conditions satisfied by both $S^{\prime}$ and $S$.

\section{Weak speedup by a subcommutative system}

7.1. Throughout this section we consider a system $\underline{B}=(B, \rightarrow)$ with normal form property, a subcommutative system $\underline{C}=(C, \rightarrow)$, and a relation $S \subseteq B \times C$, and we give conditions which are sufficient to ensure that the restriction $S_{N}$ of $S$ to $\left(B_{N} \times C\right) \cup\left(B \times C_{N}\right)$ is a speedup of $B$ by $C$. In particular the conditions ensure that $S_{N} \subseteq B_{N} \times C_{N}$. The conditions are applicable in at least one case of practical interest [6].

7.2. If the following conditions are satisfied then for all $c \in C_{N}$ and all $(b, c) \in S$ we have $b \in B_{N}$ and $(n(b), n(c)) \in S$.

(i) For ail $c \in C$ in normal form and $a$ il $(b, c) \in S, b$ is in normal form in $\underline{\underline{B}}$.

(ii) For all $c \in C$, all $(b, c) \in S$ and all contractions $c \rightarrow c$ in $\underline{\underline{C}}$, there is $b^{\prime} \in B$ such that $b^{* *} b^{\prime}$ in $\underline{B}$ and $\left(b^{\prime}, c^{\prime}\right) \in S$ (see Figure 2).

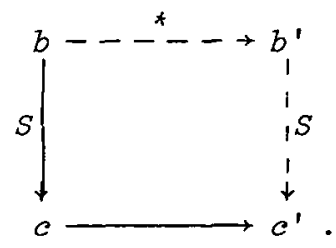

FIGURE 2

Proof. We prove by induction on $|c|$ that if $c \in C_{N}$ and $(b, c) \in S$ then $b \in B_{N}$ and $(n(b), n(c)) \in S$.

CASE 1. $|c|=0$. In this case the result follows from hypothesis (i).

CASE 2. $|c|>0$. There is $c^{\prime}$ such that $c \rightarrow_{0} c^{\prime}$, so from 
hypothesis (ii) there is $b^{\prime} \in B$ such that $b \rightarrow^{*} b^{\prime}$ and $\left(b^{\prime}, c^{\prime}\right) \in S$. Now $\left|c^{\prime}\right|=|c|-1$, so by inductive hypothesis $b^{\prime} \in B_{N}$ and $\left(n\left(b^{\prime}\right), n(c)\right) \in S$. Thus $b \in B_{N}$ and $n(b)=n\left(b^{\prime}\right)$, so we have the result.

7.3. For the remainder of this section we suppose, in addition to the hypotheses of 7.1 , that for each $b \in B$ an ordering relation $\geq_{b}$ (or briefly, $\geq$ ) is given on

$$
S(b)=\{c \in C:(b, c) \in S\}
$$

which is reflexive, antisymmetric and transitive, and is such that for all $b \in B_{N}, S(b)$ is nonempty and has a greatest element. That greatest element may be denoted $g(b)$; thus $g$ denotes a singlevalued function which is a restriction of $S$ and has domain $B_{N}$.

7.4. If the following conditions are satisfied then $\left(b, c_{1}\right) \in S$, $\left(b, c_{2}\right) \in S, c_{1} \geq c_{2}$, and $c_{1} \in C_{N}$ together imply that $c_{2} \in C_{N}$ and

$$
\left|c_{1}\right| \geq\left|c_{2}\right| \text {. }
$$

(i) For all $(b, c) \in S, b$ is in normal form in $B$ if and only if $c$ is in normal form in $C$.

(ii) $\left(b, c_{1}\right) \in S,\left(b, c_{2}\right) \in S, c_{1} \in C_{N}$, and $c_{1} \rightarrow_{0} c^{\prime}$ together imply that there are $\left(b^{\prime}, e_{1}\right),\left(b^{\prime}, e_{2}\right) \in S$ such that $e_{1} \geq e_{2}$, $c^{\prime} \rightarrow^{*} e_{1}$, and $c_{2} \rightarrow_{\leq 1} e_{2}$ (see Figure 3 ).

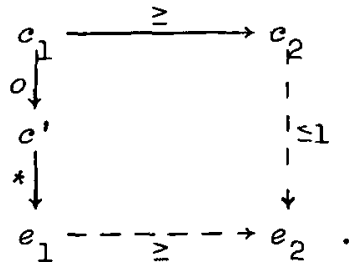

FIGURE 3

Proof. By induction on $\left|c_{1}\right|$.

CASE 1. $\left|c_{1}\right|=0$. From (i), $b$ is in normal form, so from (i) 
again so is $c_{2}$; thus $\left|c_{1}\right|=\left|c_{2}\right|=0$.

CASE 2. $\left|c_{1}\right|>0$. Then for some $e^{\prime} \in C_{N}, c_{1} \rightarrow_{0} c^{\prime}$, so from (ii) there are $\left(b^{\prime}, e_{1}\right),\left(b^{\prime}, e_{2}\right) \in S$ such that $e_{1} \geq e_{2}, c^{\prime} \rightarrow^{*} e_{1}$, and $c_{2} \rightarrow_{\leq 1} e_{2}$

Now since $c_{1} \rightarrow^{*} e_{1}$ and $\underline{\mathrm{C}}$ is subcommutative, from $3.1, e_{1} \in C_{N}$ and from 4.2 and 4.4 ,

$$
\left|e_{1}\right| \leq\left|e^{\prime}\right|=\left|c_{1}\right|-1
$$

so the inductive hypothesis applies to $e_{1}$. We conclude that $e_{2} \in c_{N}$ and $\left|e_{1}\right| \geq\left|e_{2}\right|$. As $c_{2} \rightarrow_{\leq 1} e_{2}$ it then follows that $c_{2} \in C_{N}$ and

$$
\left|c_{2}\right| \leq e_{2}+1 \leq\left|e_{1}\right|+1 \leq\left|e_{1}\right| \text {, }
$$

as required.

7.5. If the following conditions are satisfied then in the notation of 7.3, for alz $b \in B_{N}, g(b) \in C_{N}$ and $|b| \geq|g(b)|$ :

(i) as in 7.4 (i);

(ii) as in 7.4 (ii); see Figure 3;

(iii) for $a$ IL $b \in B_{N}$ and $a$ LI $b \rightarrow_{0} b^{\prime}$ there is $c^{\prime} \in C$ such that $\left(b^{\prime}, c^{\prime}\right) \in S$ and $g(b) \rightarrow_{\leq 1} c^{\prime}$ (see Figure 4$)$.

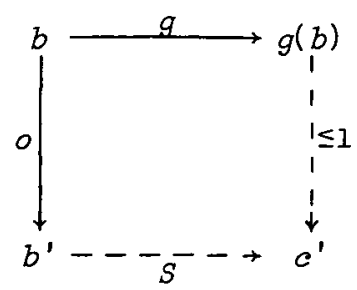

FIGURE 4

Proof. By induction on $|b|$.

CASE 1. $|b|=0$. From 7.4 (i), $g(b)$ is in normal form, so $|b|=|g(b)|=0$.

CASE 2. $|b|>0$. Although $\underline{\underline{B}}$ is not assumed subcommutative, it is 
immediate from $b \in B_{N}$ that there is some $b^{\prime} \in B_{N}$ such that $b \rightarrow_{0} b^{\prime}$ in $\underline{B}$, so that $\left|b^{\prime}\right|=|b|-1$. Thus by inductive hypothesis $g\left(b^{\prime}\right) \in C_{N}$ and $\left|g\left(b^{\prime}\right)\right| \leq\left|b^{\prime}\right|$.

From $(i i i)$ there is $c^{\prime} \in C$ such that $g(b) \rightarrow_{\leq 1} c^{\prime}$ and $\left(b^{\prime}, c^{\prime}\right) \in S$, so from $7.4, c^{\prime} \in C_{N}$, and from 7.4 and the maximality (7.3) of $g\left(b^{\prime}\right)$,

$$
\left|g\left(b^{\prime}\right)\right| \geq\left|c^{\prime}\right|
$$

$$
\text { As } \begin{aligned}
g(b) \rightarrow_{\leq 1} c^{\prime}, \quad g(b) & \in C_{N} \text { and }|g(b)| \leq\left|c^{\prime}\right|+1 \text {, so } \\
|b| & =\left|b^{\prime}\right|+1 \\
& \geq\left|g^{\prime}\left(b^{\prime}\right)\right|+1 \\
& \geq\left|c^{\prime}\right|+1 \\
& \geq|g(b)|-1+1 \\
& =|g(b)| \text { as required. }
\end{aligned}
$$

7.6. We now come to the main result of this section. We recall that $\underset{\underline{C}}{\mathrm{C}}=(C, \rightarrow)$ is assumed subcommutative, $\underset{\equiv=}{\mathrm{B}}=(B, \rightarrow)$ has the normal form property, and $S \subseteq B \times C$ has for each $b \in B$ a partial ordering $\leq$ on

$$
S(b)=\{c \in C:(b, c) \in S\}
$$

such that for all $b \in B_{N}, S(b)$ is nonempty and has a greatest element $g(b)$. Now the result is as follows.

If the following conditions are satisfied then the restriction $S_{N}$ of $S$ to $\left(B_{N} \times C\right) \cup\left(B \times C_{N}\right)$ is a subset of $B_{N} \times C_{N}$ and is a weak speedup of $\underline{B}$ by $\cong$.

(i) For all $c \in C$, alz $(b, c) \in S$, and all contractions $c+c^{\prime}$ in $\underline{\underline{C}}$, there is $b^{\prime} \in B$ such that $b \rightarrow^{*} b^{\prime}$ in $\underline{B}$ and $\left(b^{\prime}, c^{\prime}\right) \in S$ (that is, 7.4 (ii); see Figure 2).

(ii) For alz $(b, c) \in S, b$ is in normal form in $\mathrm{B}$ if and only if $c$ is in normal form in $\underline{\underline{c}}$ (that is, 7.4 (i)).

(iii) $\left(b, c_{1}\right) \in S,\left(b, c_{2}\right) \in S, c_{1} \in C_{N}, c_{1} \geq c_{2}$, and $c_{1} \rightarrow_{0} e^{\prime}$ together imply that there are $\left(b^{\prime}, e_{1}\right),\left(b^{\prime}, e_{2}\right) \in S$ such that 
$e_{1} \geq e_{2}, \quad c^{\prime} \rightarrow * e_{1}$, and $e_{2} t_{\leq 1} e_{2}$ (that is, 7.4 (ii); see Figure 3).

(iv) For $a$ ll $b \in B_{N}$ and $a$ ll $b \rightarrow_{0} b^{\prime}$ there is $c^{\prime} \in C$ such that $\left(b^{\prime}, c^{\prime}\right) \in S$ and $g(b) \rightarrow_{\leq 1} c^{\prime}$ (that is, 7.5 (iii); see Figure 4).

Proof. It follows from 7.4 and 7.5 that if $b \in B_{N}$ and $(b, c) \in S$, then $g(b), c \in C_{N}$ and $|g(b)| \geq|c|$. If conversely $c \in C_{D V}$ and $(b, c) \in S$, it follows from 7.2 that $b \in B_{N}$. Thus $S_{N} \subseteq B_{N} \times C_{N}$, as required for a weak normal representation.

Further, since for all $b \in B_{N},(b, g(b)) \in S$, then $6.2(i)$ is satisfied by $S_{N}$. Since $S_{N} \subseteq B_{N} \times C_{N}, 7.2$ shows that for all $(b, c) \in S_{N}, n(b), n(c) \in S_{N}$, as required for 6.2 (ii). Hence $S_{N}$ is a weak normal representation of $\underline{B}$ by $\underline{\mathrm{C}}$.

Finally, for all $b \in B_{N},|b| \geq|g(b)|$, from 7.5 , and as noted above $|g(b)| \geq|c|$ for all $(b, c) \in S_{N}$, so we conclude, as required by the definition of weak speedup, that $|b| \geq|c|$.

\section{Weak speedup of one subcommutative system by another}

8.1. In this short section we give a simplified weak speedup result which is applicable when the system $\underline{\underline{B}}=(B, \rightarrow)$ to be speeded up is also. subcommutative.

As at the beginning of the previous section we assume that $\underline{\mathrm{C}}=(C, \rightarrow)$ is a subcommutative system and that $S \subseteq B \times C$, and we give conditions which are sufficient to ensure that $S_{N}=S \cap\left(\left(B_{N} \times C\right) \cup\left(B \times C_{N}\right)\right)$ is a weak speedup of $\underline{\underline{B}}$ by $\stackrel{C}{\underline{C}}$. The main lemmas are 7.2 and the following modification of 7.4 .

8.2. If the following conditions are satisfied, then for all $b \in B_{N}$ and all $c \in C$ such that $(b, c) \in S$ we have $c \in C_{N}$ and $|b| \geq|c|$.

(i) If $c \in C,(b, c) \in S$ and $b$ is in normal form, then $c$ is in normal form.

(ii) If $c \in C,(b, c) \in S, b \in B_{N}$, and $b \rightarrow_{0} b^{\prime}$ in $\underline{\underline{B}}$, then 
there is $b^{\prime \prime} \in B$ and $e \in C$ such that $b^{\prime} \rightarrow^{*} b^{\prime \prime}$ in $B,\left(b^{\prime \prime}, e\right) \in S$, and $c t_{\leq 1} e$ in $C$ (see Figure 5)

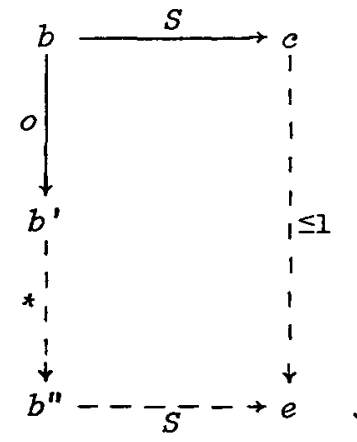

FIGURE 5

Proof. By induction on $|b|$.

CASE 1. $|b|=0$. Immediate from (i).

CASE 2. $|b|>0$. For some $b^{\prime} \in B_{N}, b \rightarrow_{0} b^{\prime}$, so $\left|b^{\prime}\right|=|b|-1$, and from $(i i), b^{\prime} \rightarrow b^{\prime \prime}$ where $\left(b^{\prime \prime}, e\right) \in S$ and $c \rightarrow_{\leq I} e$. Thus $b^{\prime \prime} \in B_{N}$, from 3.1 , and $\left|b^{\prime \prime}\right| \leq\left|b^{\prime}\right|<|b|$, so by inductive hypothesis $e \in C_{N}$. As $e \rightarrow_{\leq 1} e$, then $e \in C_{N}$, and

$$
\begin{aligned}
|c| & \leq|e|+1 \\
& \leq\left|b^{\prime \prime}\right|+1 \text { by inductive hypothesis } \\
& \leq\left|b^{\prime}\right|+1 \text { from } 4.2 \text { and } 4.4 \\
& =|b| \text { as required. }
\end{aligned}
$$

8.3. Hence we have the main result of this section, as follows.

For $\underline{B}, \underline{\mathrm{C}}, S, S_{N}$ as in 8.1, if the following conditions are satisfied, then $S_{N} \subseteq B_{N} \times C_{N}$ and $S_{N}$ is a weak speedup of $\underline{\underline{\mathrm{B}}}$ by $\underline{\mathrm{C}}$.

(i) For all $b \in B_{N}$ there is $(b, e) \in S$.

(ii) For all $(b, c) \in S, b$ is in normal form in $\underline{B}$ if and only if $c$ is in normal form in $\underline{\underline{C}}$ (that is, 7.2 (i) and 8.2 (i)).

(iii) For all $c \in C$, all $(b, c) \in S$, and all contractions $c \rightarrow c$ ' in $\subseteq$, there is $b^{\prime} \in B$ such that $b+b^{\prime}$ in $B$ and $\left(b^{\prime}, c^{\prime}\right) \in S$ (that is, 7.2 (ii); see Figure 2). 
(iv) If $c \in C,(b, c) \in S, b \in B_{N}$, and $b \rightarrow_{0} b^{\prime}$ in $\underline{\underline{B}}$, then there is $b^{\prime \prime} \in B$ and $e \in C$ such that $b^{\prime} \rightarrow^{*} b^{\prime \prime}$ in $\underline{\underline{B}},\left(b^{\prime \prime}, e\right) \in S$, and $c \rightarrow_{\leq 1} e$ in $C$ (that is, 8.2 (iii); see Figure 5).

Proof. If $(b, c) \in S$ and $b \in B_{N}$ then $c \in C_{N}$ from 8.2. If conversely $(b, c) \in S$ and $c \in C_{N}$ then $b \in B_{N}$ from 7.2 , so $S_{N} \subseteq B_{N} \times C_{N}$

Hence, and in view of condition (i) $6.2(i)$ is satisfied. Also 6.2 (ii) is given by 7.2 , and 8.2 provides the inequality which is required by 6.5 to complete the verification that $S_{N}$ is a weak speedup of $\underline{\underline{B}}$ by $\underline{\underline{C}}$.

\section{Discussion}

9.1. The term "dumny contraction", used in a specific sense in Section 4 , has also been used in [5] in an abstract sense. The concept of Section 4 is an example of the abstract notion of [5] for subcommutative systems, but not in general.

9.2. The hypotheses of 5.2 can be sharpened if one allows the mention of o-contractions. The point however is that the hypotheses of 5.2 do not mention them; for $a, b \in B$, membership by $(a, b)$ of $\overrightarrow{ }_{o}$ is generally difficult to decide in practice, even given that $a \rightarrow b$ and the information that $a \in B_{N}$.

9.3. Although we have defined speedup and strong speedup in Section 6 , the conditions of Sections 7 and 8 lead only to weak speedups. The additional restrictions 6.3 and 6.4 which strengthen weak speedup to speedup and strong speedup are relatively elementary and may be left for verification as required in particular applications.

\section{References}

[1] Hartmut Ehrig and Barry K. Rosen, "Commutativity of independent transformations on complex objects" (IBM Research Report RC 6251 (\#26882), New York, 1976). 
[2] G. Pacini, "An optimal fix-point computation rule for a simple recursive language" (Nota Interna B73-10, Pisa, 1973).

[3] G. Pacini, C. Montangero, F. Turini, "Graph representation and computation rules for typeless recursive languages", Automata, languages and programing, 157-169 (2nd Colloquium, University of Saarbrücken, 1974. Lecture Notes in Computer Science, 14. Springer-Verlag, Berlin, Heidelberg, New York, 1974).

[4] Barry K. Rosen, "Tree-manipulating systems and Church-Rosser theorems", J. Assoc. Comput. Mach. 20 (1973), 160-187.

[5] John Staples, "Optimal reduction in replacement systems", Buzl. Austral. Math. Soc. 16 (1977), 341-349.

[6] John Staples, "Speeding up subtree replacement systems", submitted.

[7] John Staples, "Optimal evaluations of nonlinear expressions", submitted.

[8] Jean Vuillemin, "Correct and optimal implementations of recursion in a simple programming language", Proc. Fifth Annual ACM Sympos. Theory of Computing, 224-259 (Austin, Texas, 1973).

[9] Christopher Peter Wadsworth, "Semantics and pragmatics of the lamdacalculus" ( $\mathrm{PhD}$ thesis, University of Oxford, Oxford, 1971).

Department of Mathematics and Computer Science, Queensland Institute of Technology, Brisbane, Queensland. 